\title{
Convulsion on table in A ASA I Pregnant patient posted for emergency caesarean section: anesthesia
} management

\begin{abstract}
Convulsions occurring on the operating table in any patient, especially a pregnant patient, can be a nightmare. Initial management with regard to airway, breathing and anticonvulsants remains same. The differential diagnosisinclude eclampsia, hypoglycaemia, peripartum cerebrovascular thrombosis, subarachnoid haemorrhage, infection, intracranial tumour, head injury, idiopathic epilepsy, vasculitis, amniotic fluid embolism (AFE), etc. MRI brain of our patient revealed posterior reversible encephalopathy syndrome (PRES), a neuro-radiological diagnosis. Management is supportive with anticonvulsants, cerebral decongestants and treating the underlying cause including blood pressure control and early caesarean section. There is a small risk of permanent neurological damage or death.
\end{abstract}

Keywords: caesarean section, convulsions, posterior reversible encephalopathy syndrome, eclampsia, phenytoin
Volume 5 Issue I - 2016

\author{
Wajekar Anjana S,' Manisha Taware,' Uma \\ Kamat $^{2}$ \\ 'Assistant Professor, King Edward Memorial Hospital, India \\ ${ }^{2}$ Department of Anaesthesia, King Edward Memorial Hospital, \\ India \\ Correspondence: Anjana SWajekar,Assistant Professor, \\ Department of Anaesthesia, King Edward Memorial Hospital, \\ Mumbai, India, Tel +91-9930I04I06, \\ Email anjanawajekar@gmail.com
}

Received: October 27, 2016 | Published: June 0I, 2016
Abbreviations: AFE: amniotic fluid embolism; PRES: posterior reversible encephalopathy syndrome; ASA: American society of anaesthesiologists; G2P1L1: gravida 2 para 1 live birth 1

\section{Introduction}

Peripartum convulsion can significantly increase both maternal and foetal morbidity and mortality. ${ }^{1}$ Any convulsion occurring in a pregnant patient after 20 weeks is always assumed to be eclampsia and treated likewise. Immediate termination of pregnancy is advised only in case of recurrent seizures in labour. ${ }^{1}$ But every seizure increases the risk of abruption and maternal aspiration and fetal acidosis, hypoxia, intracranial bleed and death. ${ }^{1}$ Other differential diagnosis of peripartum seizure also needs to be ruled out but initial management of convulsion remains airway, breathing and circulation, seizure control and fetal health. We present a case of management of preoperative on table convulsions in an American Society of Anaesthesiologists (ASA) grade I patient posted for emergency caesarean section.

\section{Case presentation}

A 22 year old, G2P1L1 patient with history of one previous caesarean section, was taken for emergency caesarean section for non-reassuring foetal Doppler. Her systemic examination and all her routine blood investigations including complete blood count, renal and liver function test and coagulation profile were normal. In the operating room, her pulse was $56 / \mathrm{min}$, blood pressure $178 / 108 \mathrm{~mm}$ of hg and room air saturation $100 \%$. Left uterine displacement was maintained. Ringer's lactate was started. Before the patient could be positioned for spinal anaesthesia, patient developed left hand focal convulsion with secondary generalisation which lasted for 2-3 minutes, followed by unconsciousness. Midazolam $1 \mathrm{mg}$ was injected. The patient was immediately incubated following rapid sequence induction with thiopentone $250 \mathrm{mg}$ and succinylcholine $100 \mathrm{mg}$. Antiaspiration prophylaxis with ranitidine $50 \mathrm{mg}$ and ondansetron $4 \mathrm{mg}$ was given. Anaesthesia was maintained with isoflurane 0.8MAC in oxygen: nitrous oxide $(50 \%)$ and intermittent vecuronium bolus. Her blood sugar level was $81 \mathrm{mg} / \mathrm{dl}$. Labetalol $20 \mathrm{mg}$ was given for persistent hypertension intra operatively. The baby was delivered in 10 minutes with an Apgar score 9 at $1 \mathrm{~min}$. Thereafter fentanyl $100 \mathrm{mcg}$ and oxytocin $20 \mathrm{U}$ in slow drip were given. The uterus was well contracted and haemostasis achieved. Levetiracetam 500mg was administered. The patient was extubated on table after return of spontaneous ventilation and full consciousness. Patient was shifted to ICU. Hypertension persisted postoperatively which was treated with bolus dose of labetalol 10mg and later shifted to oral alphamethyldopa $500 \mathrm{mg}$ and nifedepine $10 \mathrm{mg}$. The patient had 6 more episodes of generalised tonic clonic convulsions within the next 24 hours. Phenytoin $100 \mathrm{mg}$, mannitol and dexamethasone were added. Postoperative proteinuria was negative. All other postoperative investigations remained normal. Fundoscopy revealed no papilledema. Postoperative MRI brain showed bilateral hyper densities in cerebellar hemispheres, basal ganglia, occipital, parietal, temporal and frontal lobes consistent with posterior reversible encephalopathy syndrome (PRES). She was discharged on day 15 with antihypertensive and anticonvulsant medications.

\section{Discussion}

The differential diagnosis of peripartum seizures includes eclampsia, hypoglycaemia, peripartum cerebrovascular thrombosis, subarachnoid haemorrhage, infection (meningitis or encephalitis), intracranial tumour, head injury, idiopathic epilepsy, vasculitis, amniotic fluid embolism (AFE), idiopathic, psychogenic, etc. ${ }^{2}$ Our patient had no past history of epilepsy, head trauma or fever. Her white cell counts and sugar levels were normal. AFE is generally associated with cardiopulmonary collapse and coagulopathy. ${ }^{2}$ MRI brain was diagnostic and showed signs consistent with PRES

Rapid control of maternal hypertension with labetalol is important. Rapid institution of general anaesthesia with endotracheal intubation was the best course of action, since the patient was taken up in view of non-reassuring fetal Doppler and further delay was unadvisable. It is preferable to avoid magnesium sulphate intra operatively due to prolongation of non-depolarising blockade and uterine atony. ${ }^{3,4}$ Neonatal exposure to antiepileptic drugs preoperatively may adversely affect their neuronal development. Forcelli and coworkers found that synaptic maturation was absent in rats exposed to a single dose of 
phenobarbital, phenytoin, or lamotrigine but not levetiracetam. ${ }^{5}$ Studies have found in utero exposure of phenytoin or levetiracetam has a very low risk of congenital malformations $(<3 \%)$ and it is dose dependant. ${ }^{6,7}$ In view of potential foetal complications, presence of paediatrician during delivery is essential as foetal resuscitation may be required.

Posterior reversible encephalopathy syndrome (PRES) is a neuro-radiological diagnosis, first described by Hinchey et al.$^{8}$ It is recognised by a variety of names like reversible posterior cerebral edema syndrome, reversible posterior leukoencephalopathy syndrome and reversible occipital parietal encephalopathy. It is characterised by vague symptoms like headache, altered sensorium, visual disturbances, vomiting and seizures especially with secondary generalization. ${ }^{8}$ Although acute hypertension is not a symptom of PRES but it is found in $67 \%$ of the patients with PRES. ${ }^{8}$ It can be associated with a wide array of diseases like preeclampsia, HELLP syndrome, encephalopathy, renal diseases, cerebral infections, sepsis, immuno suppressants, etc. ${ }^{8,9}$ The pathophysiology is generally intracranial vasogenicedema secondary to cerebral hyper perfusion. ${ }^{8,9}$ MRI brain with T2 fluid attenuated inversion recovery (FLAIR) sequence is diagnostic and showed a typical holo hemispheric watershed pattern in our patient. Management is supportive with anticonvulsants, cerebral decongestants and treating the underlying cause including blood pressure control and early caesarean section. ${ }^{9}$ There is a small risk of permanent neurological damage or death. ${ }^{8}$

Basic management of on table convulsion with regard to airway, breathing and circulation and anticonvulsants should always be kept in mind and management best titrated to maternal and foetal outcome should be done.

\section{Acknowledgments}

None.

\section{Conflicts of interest}

Author declare that there is no conflict of interest.

\section{Funding}

None.

\section{References}

1. Pandey R, Garg R, Darlong V, et al. Recurrent seizures in pregnancyepilepsy or eclampsia:a diagnostic dilemma? A case report. AANA $J$. 2011;79(5):388-390.

2. Sprung J, Rakic M, Patel S. Amniotic fluid embolism during epidural anesthesia for cesarean section. Acta Anaesthesiol Belg. 1991;42(4):225231.

3. Habe K, Kawasaki T, Sata T. A case of prolongation of rocuronium neuromuscular blockade in a pregnant patient receiving magnesium. Masui. 2014;63(7):817-819.

4. Siddik-Ahmad RI, Garabedian MJ, et al. Inadequate uterine contractions increase risk for uterine atony at cesarean delivery. Obstet Gynecol. 2014;123 Suppl:80S.

5. Forcelli PA, Janssen MJ, Vicini S, et al. Neonatal exposure to antiepileptic drugs disrupts striatal synaptic development. Ann Neurol 2012;72(3):363-372.

6. Harden CL. Pregnancy and epilepsy. Continuum (MinneapMinn). 2014;20:60-79.

7. Hernández Díaz S, Smith CR, Shen A, et al. Comparative safety of antiepileptic drugs during pregnancy. Neurology. 2012;78(21):16921699.

8. Postma IR, Slager S, Kremer HPH, et al. Long-term consequences of the posterior reversible encephalopathy syndrome in eclampsia and preeclampsia:a review of the obstetric and nonobstetric literature. Obstet Gynecol Surv. 2014;69(5):287-300.

9. Fukami T, Asakura H, Takeshita T. Reversible posterior leukoencephalopathy syndrome due to eclampsia in a woman with a twin pregnancy produced with donated oocytes. J Nippon Med Sch. 2013;80(3):230-233. 